\title{
SEQUENTIAL LAMINATES IN MULTIPLE-STATE OPTIMAL DESIGN PROBLEMS
}

NENAD ANTONIĆ AND MARKO VRDOLJAK

Received 23 November 2004; Revised 2 March 2005; Accepted 4 April 2005

In the study of optimal design related to stationary diffusion problems with multiple-state equations, the description of the set $\mathscr{H}=\left\{\left(\mathbf{A a}_{1}, \ldots, \mathbf{A} \mathbf{a}_{m}\right): \mathbf{A} \in \mathscr{K}(\theta)\right\}$ for given vectors $\mathbf{a}_{1}, \ldots, \mathbf{a}_{m} \in \mathbb{R}^{d}(m<d)$ is crucial. $\mathscr{K}(\theta)$ denotes all composite materials (in the sense of homogenisation theory) with given local proportion $\theta$ of the first material. We prove that the boundary of $\mathscr{H}$ is attained by sequential laminates of rank at most $m$ with matrix phase $\alpha \mathbf{I}$ and core $\beta \mathbf{I}(\alpha<\beta)$. Examples showing that the information on the rank of optimal laminate cannot be improved, as well as the fact that sequential laminates with matrix phase $\alpha \mathbf{I}$ are preferred to those with matrix phase $\beta \mathbf{I}$, are presented. This result can significantly reduce the complexity of optimality conditions, with obvious impact on numerical treatment, as demonstrated in a simple numerical example.

Copyright (c) 2006 N. Antonić and M. Vrdoljak. This is an open access article distributed under the Creative Commons Attribution License, which permits unrestricted use, distribution, and reproduction in any medium, provided the original work is properly cited.

\section{Introduction}

In optimal design problems, the goal is to determine the optimal distribution of given materials in a given domain. Optimality of a distribution is measured by an objective function, which is usually an integral functional depending on the distribution of materials and the state function, obtained as a solution of the associated boundary value problem for the corresponding partial differential equation. For example, in the thermal conductivity problem, the state function $u$ is the temperature of the considered body $\Omega \subseteq \mathbb{R}^{d}$, the solution of boundary value problem

$$
\begin{gathered}
-\operatorname{div}(\mathbf{A} \nabla u)=f, \\
u \in \mathrm{H}_{0}^{1}(\Omega),
\end{gathered}
$$

for some given source term $f \in \mathrm{H}^{-1}(\Omega)$. The conductivity A reflects the distribution of given materials. There is abundant literature in this field $[1,6,8,11,12]$. 


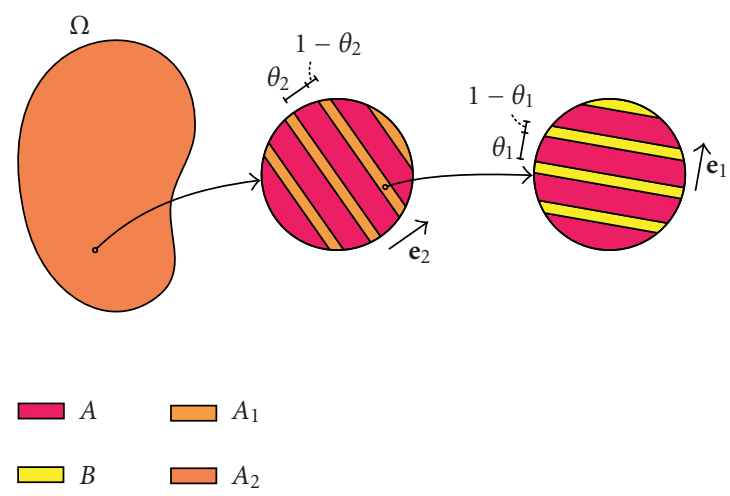

Figure 1.1. $\mathbf{A}_{2}$ is the rank-two laminate belonging to $\mathscr{K}(\theta)$ with $\theta=1-\left(1-\theta_{1}\right)\left(1-\theta_{2}\right)$ and $\kappa_{1}=\theta_{1}$, $\kappa_{2}=\theta_{2}\left(1-\theta_{1}\right) / \theta$ in $(1.2)$.

Practical significance of such problems can be envisaged from the following example. Imagine a device in which heat is being generated, and for which proper operation has to be kept bellow certain temperature. If the geometry of the radiator is given, the goal is to arrange the materials for its construction so as to satisfy the temperature constraints, while at the same time keep the cost low. If we are given two conducting materials, one good (and expensive) and one poorer, the question is where to put the small quantity of good one in order to maximise the cooling effect.

Such problems of optimal design are known to be ill-posed: there is no optimal solution, that is, the smallest possible value of the objective function is not achieved among simple mixtures. Therefore, a relaxation is required, introducing generalised designs, described by the theory of homogenisation as an H-closure (or, equivalently, a G-closure, since we are dealing with symmetric coefficients) of the original set of mixtures. However, the $\mathrm{H}$-closure is known only in a few particular cases. Anyway, the structure of this closure is well known (see [12]): for a given local proportion of materials, this set is related only to the eigenvalues of conductivity matrix (for this set, we will use the notation $\mathscr{K}(\theta)$, where $\theta$ is the proportion of the first material).

A particularly simple mixture can be obtained by lamination; we interchange the layers of two materials, so that their thickness ratio is $\theta /(1-\theta)$. Not only such materials are amenable to technological production, but their properties can be easily computed. If we pass to the zero limit of layer thickness (with the ratio preserved), we obtain a homogenised material with constant coefficients, whose properties are close to those of rapidly changing (and thus numerically intractable) fine layers.

This process can be continued; in the second step, we can layer the first material with the earlier described mixture in order to obtain a rank-two laminate. If we imagine a fine mixture obtained in such a way in the domain $\Omega$, when we look through a microscope first at one magnification, and later at a finer magnification, we get Figure 1.1. The precise description and formulae for the coefficients are given by the following definition.

A rank- $m$ sequential laminate (introduced in $[10]$, see also $[1,12]$ ) $\mathbf{A}^{*}$ with matrix phase $\mathbf{A}$ and core $\mathbf{B}$ in proportions $\theta$ and $1-\theta$, respectively, is described by $m$ vectors $\mathbf{e}_{1}, \ldots, \mathbf{e}_{m}$ (the directions of successive laminations) and nonnegative parameters $\kappa_{1}, \ldots, \kappa_{m}$ 
whose sum equals 1 , via the formula

$$
(1-\theta)\left(\mathbf{A}^{*}-\mathbf{A}\right)^{-1}=(\mathbf{B}-\mathbf{A})^{-1}+\theta \sum_{i=1}^{m} \kappa_{i} \frac{\mathbf{e}_{i} \otimes \mathbf{e}_{i}}{\mathbf{A e}_{i} \cdot \mathbf{e}_{i}}
$$

Similarly, a rank- $m$ sequential laminate $\mathbf{A}^{*}$ with matrix $\mathbf{B}$ and core $\mathbf{A}$ is defined by

$$
\theta\left(\mathbf{A}^{*}-\mathbf{B}\right)^{-1}=(\mathbf{A}-\mathbf{B})^{-1}+(1-\theta) \sum_{i=1}^{m} \kappa_{i} \frac{\mathbf{e}_{i} \otimes \mathbf{e}_{i}}{\mathbf{B} \mathbf{e}_{i} \cdot \mathbf{e}_{i}}
$$

In this paper, we discuss only the case of two isotropic materials with conductivities $\alpha$ and $\beta(\alpha<\beta)$. If a local proportion of the first material is $\theta$, then effective materials (obtained by the process of homogenisation) consist of matrices with eigenvalues satisfying [10]

$$
\begin{gathered}
\lambda_{j} \leq \lambda_{\theta}^{+} \quad(j=1, \ldots, d), \\
\sum_{j=1}^{d} \frac{1}{\lambda_{j}-\alpha} \leq \frac{1}{\lambda_{\theta}^{-}-\alpha}+\frac{d-1}{\lambda_{\theta}^{+}-\alpha}, \\
\sum_{j=1}^{d} \frac{1}{\beta-\lambda_{j}} \leq \frac{1}{\beta-\lambda_{\theta}^{-}}+\frac{d-1}{\beta-\lambda_{\theta}^{+}},
\end{gathered}
$$

where $\lambda_{\theta}^{-}=(\theta / \alpha+(1-\theta / \beta))^{-1}$ and $\lambda_{\theta}^{+}=\theta \alpha+(1-\theta) \beta$. In two- and three-dimensional cases, this set is depicted in Figure 1.2. In (1.4), we omit the constraints $\lambda_{j} \geq \lambda_{\theta}^{-}, j=$ $1, \ldots, d$ since they follow from other inequalities [1, Remark 2.2.14].

Remark 1.1. In the case of isotropic materials, it easily follows that the rank- $m$ laminate has $d-m$ eigenvalues equal to $\lambda_{\theta}^{+}$and satisfies (1.5) if the matrix material is $\alpha \mathbf{I}$, or (1.6) if the matrix material is $\beta \mathbf{I}$. Equalities in (1.5) and (1.6) hold simultaneously only in the case of first-order laminates, where $m-1$ eigenvalues are equal to $\lambda_{\theta}^{+}$and one is $\lambda_{\theta}^{-}$.

An optimal design problem for (1.1) with two isotropic materials is studied in [8]. The method used there actually relies on the following lemma.

Lemma 1.2. For $d \geq 2, \theta \in[0,1]$, and $\mathbf{e} \in \mathbb{R}^{d}$, the $\operatorname{set} \mathscr{K}(\theta) \mathbf{e}$ is the closed ball determined by its diameter segment $\left[\lambda_{\theta}^{-} \mathbf{e}, \lambda_{\theta}^{+} \mathbf{e}\right]$. The boundary (sphere) is attained by rank-one laminates.

This result is true even in the case of mixing any number of anisotropic materials [11], where the same method for optimal design is successfully applied in spite of not knowing the characterisation of the H-closure.

\section{Multiple-state equations}

In the above example, imagine that the radiator has to work well not only in one regime of operation, but in at least two. The difficulty is that the obtained optimal distribution will change if $f$ is changed. This situation refers to multistate optimal design problems, where the optimality takes into account temperatures $u_{1}, \ldots, u_{m}$ obtained for $f_{1}, \ldots, f_{m}$, 
4 Multiple-state optimal design problems

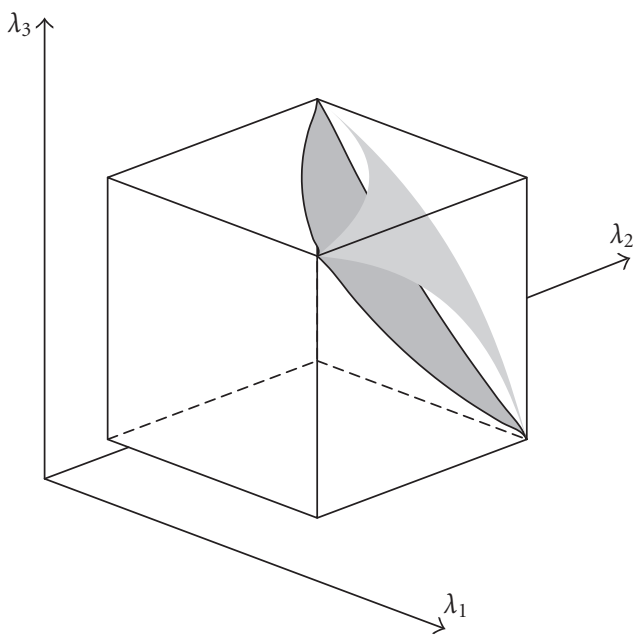

(a)

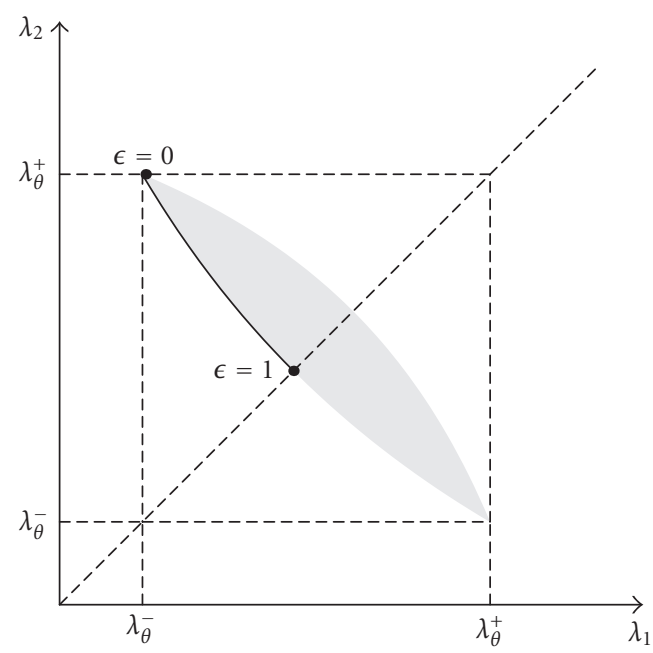

(b)

Figure 1.2. $\mathscr{K}(\theta)$ consists of symmetric matrices with eigenvalues (a) inside cube and between two hyperboloid surfaces for $d=3$ or (b) inside shaded region for $d=2$.

respectively [1, Section 3.1.3]. The available results for this type of problems are rather scarce, with practical calculations performed only in two-dimensional cases.

Let us now make the above ideas more precise. The conductivity of the mixture of two isotropic materials with conductivities $\alpha \mathbf{I}$ and $\beta \mathbf{I}$ can be written as

$$
\mathbf{A}(\mathbf{x})=\chi(\mathbf{x}) \alpha \mathbf{I}+(1-\chi(\mathbf{x})) \beta \mathbf{I},
$$


where $\chi: \Omega \rightarrow\{0,1\}$ denotes the characteristic function of the first material. For given $\mathbf{A}$, the corresponding temperatures $u_{1}, \ldots, u_{m}$ are determined by

$$
\begin{gathered}
-\operatorname{div}\left(\mathbf{A} \nabla u_{i}\right)=f_{i}, \\
u_{i} \in \mathrm{H}_{0}^{1}(\Omega) .
\end{gathered}
$$

With notation $\mathbf{u}=\left(u_{1}, \ldots, u_{m}\right)$, the optimality of a given mixture is measured by the cost functional

$$
J(\chi)=\int_{\Omega}\left(\chi(\mathbf{x}) g_{\alpha}(\mathbf{x}, \mathbf{u}(\mathbf{x}))+(1-\chi(\mathbf{x})) g_{\beta}(\mathbf{x}, \mathbf{u}(\mathbf{x}))\right) d \mathbf{x}
$$

and our goal is to minimise this functional over the set of all measurable characteristic functions $\chi$ on $\Omega$. Functions $g_{\alpha}$ and $g_{\beta}$ should be chosen such that $\mathbf{u} \mapsto\left(g_{\alpha}(\cdot, \mathbf{u})\right.$, $\left.g_{\beta}(\cdot, \mathbf{u})\right)$ is continuous from $\mathrm{H}_{0}^{1}\left(\Omega, \mathbb{R}^{m}\right)$ weak into $\mathrm{L}^{2}\left(\Omega, \mathbb{R}^{2}\right)$ strong $[11]$.

As mentioned in the introduction, the relaxation of this problem is necessary. It consists in introducing a local proportion $\theta: \Omega \rightarrow[0,1]$ of material $\alpha \mathbf{I}$, instead of $\chi$ with values either 0 or 1 . Now, from the homogenisation theory, it is known that (2.1) cannot be used to express $\mathbf{A}$ in terms of $\theta$, since we have to accept all possible conductivities belonging to the set $\mathscr{K}(\theta)$. The relaxation is written as

$$
\begin{gathered}
J(\theta, \mathbf{A})=\int_{\Omega}\left(\theta(\mathbf{x}) g_{\alpha}(\mathbf{x}, \mathbf{u}(\mathbf{x}))+(1-\theta(\mathbf{x})) g_{\beta}(\mathbf{x}, \mathbf{u}(\mathbf{x}))\right) d \mathbf{x} \longrightarrow \min , \\
\theta \in \mathrm{L}^{\infty}(\Omega,[0,1]), \quad \mathbf{A} \in \mathcal{K}(\theta) \quad \text { a.e. on } \Omega \text { where } u_{i} \text { are solutions of }(2.2) .
\end{gathered}
$$

To apply the same idea, as for the optimal design with one-state equation that was presented in $[8,11]$, the first step is to describe the set

$$
\mathscr{H}=\left\{\left(\mathbf{A a}_{1}, \ldots, \mathbf{A a}_{m}\right): \mathbf{A} \in \mathscr{K}(\theta)\right\}
$$

for given vectors $\mathbf{a}_{1}, \ldots, \mathbf{a}_{m} \in \mathbb{R}^{d}$. In the special case $d=3$ and $m=2$, we obtained a parametrisation of this set in $[2,3]$, which could be used to visualise it, using a computer program. Analysing a number of examples, we noticed an interesting behaviour on the boundary, which we prove in this paper.

Theorem 2.1. For any linearly independent set $\left\{\mathbf{a}_{1}, \ldots, \mathbf{a}_{m}\right\}$ of vectors in $\mathbb{R}^{d}$ such that $m<d$, the boundary of the set

$$
\mathscr{H}=\left\{\left(\mathbf{A a}_{1}, \ldots, \mathbf{A a}_{m}\right): \mathbf{A} \in \mathscr{K}(\theta)\right\}
$$

is attained by sequential laminates of rank at most $m$ with matrix phase $\alpha \mathbf{I}$.

Remark 2.2. For the case of linearly dependent set $\left\{\mathbf{a}_{1}, \ldots, \mathbf{a}_{m}\right\}$, the result of the theorem is true if $\tilde{m}=\operatorname{dim}\left(\operatorname{Span}\left(\left\{\mathbf{a}_{1}, \ldots, \mathbf{a}_{m}\right\}\right)\right)<d(m$ could be even greater than $d)$. In this case, the boundary of $\mathscr{H}$ is attained by sequential laminates of rank at most $\tilde{m}$ with matrix phase $\alpha \mathrm{I}$. This fact follows easily from the beginning of Section 4 .

The problem we are dealing with originates from [12], where it is shown that in the case of mixtures of anisotropic materials, the set $\mathcal{H}$ is convex. We wish to thank professor Tartar for bringing this problem to our attention. 


\section{Normal cones for $\Lambda(\theta)$}

In the proof of Theorem 2.1, it will be shown that the optimisation of a linear function over the set of matrices $\mathscr{K}(\theta)$ can be reduced to optimisation of a related linear function over the set of corresponding eigenvalues $\Lambda(\theta)$ described by (1.4)-(1.6). The solution of the latter problem can be expressed using the notion of normal cone.

In the case of a convex set $C \subseteq \mathbb{R}^{d}$, the definition of normal cone $\mathcal{N}_{C}(\mathbf{x})$ at a point $\mathbf{x} \in C$ is simple (see [9]):

$$
\mathcal{N}_{C}(\mathbf{x})=\left\{\boldsymbol{\xi} \in \mathbb{R}^{d}:(\forall \mathbf{y} \in C) \boldsymbol{\xi} \cdot(\mathbf{y}-\mathbf{x}) \leq 0\right\}
$$

wherefrom it easily follows that $\mathcal{N}_{C}(\mathbf{x})$ is a closed convex cone. Further properties of normal cones and some generalisations can be found in [7]. For some sets, the normal cones can easily be computed, for example, if $C$ is a set of the form

$$
C=\left\{\mathbf{x} \in \mathbb{R}^{d}: f_{j}(\mathbf{x}) \leq 0, j=1, \ldots, n\right\}
$$

for given differentiable convex functions $f_{j}$ satisfying the Slater condition

$$
(\exists \tilde{\mathbf{x}} \in C)(\forall j \in\{1, \ldots, n\}), \quad f_{j}(\tilde{\mathbf{x}})<0 .
$$

If $\mathbf{x}$ belongs to the boundary of $C$, so we have equalities $f_{j}(\mathbf{x})=0, j \in J \subseteq\{1, \ldots, n\}$, then [9, Theorem 23.7, Corollary 23.8.1] implies that

$$
\begin{aligned}
\mathcal{N}_{C}(\mathbf{x}) & =\left\{\sum_{j \in J} \lambda_{j} \nabla f_{j}(\mathbf{x}): \lambda_{j} \geq 0, j \in J\right\} \\
& =\left\{\sum_{j=1}^{n} \lambda_{j} \nabla f_{j}(\mathbf{x}): \lambda_{j} \geq 0, \lambda_{j} f_{j}(\mathbf{x})=0, j=1, \ldots, n\right\} .
\end{aligned}
$$

If the minimising function is convex over the admissible convex set, the usual necessary conditions of optimality are also sufficient. More precisely, if $C \subseteq \mathbb{R}^{d}$ is a convex set and $f: C \rightarrow \mathbb{R}$ is convex and differentiable in $\mathbf{x} \in C$, then $\mathbf{x}$ is a minimiser for $f$ over $C$ if and only if $-\nabla f(\mathbf{x})$ belongs to the normal cone $\mathcal{N}_{C}(\mathbf{x})[9$, Theorem 27.4].

The above results are also valid in the case of nonsmooth functions, where the subdifferential plays the role of the gradient (see $[7,9])$. Now we will write down a description of normal cones for set $\Lambda(\theta)$, which will be used in the next section. 
Lemma 3.1. Let $\lambda$ belong to the boundary of set $\Lambda(\theta)$ given by (1.4)-(1.6). We have the following mutually excluding possibilities.

(I) Both inequalities in (1.5) and (1.6) are strict, and the equalities hold in (1.4) for some set $J \neq \varnothing$ of indices $j$. Then,

$$
\mathcal{N}_{\boldsymbol{\Lambda}(\theta)}(\boldsymbol{\lambda})=\left\{\mathbf{k} \in[0,+\infty\rangle^{d}: k_{j}=0 \text { for } j \notin J\right\} .
$$

(II) There exist equalities only in (1.4) for indices $j \in J$ (possibly empty) and (1.5). Then,

$$
\begin{aligned}
\mathcal{N}_{\boldsymbol{\Lambda}(\theta)}(\boldsymbol{\lambda})=\left\{\mathbf{k} \in \mathbb{R}^{d}: k_{j}=-\frac{\eta_{\alpha}}{\left(\lambda_{j}-\alpha\right)^{2}} \text { for } j \notin J,\right. \\
\left.k_{j}=-\frac{\eta_{\alpha}}{\left(\lambda_{\theta}^{+}-\alpha\right)^{2}}+\eta_{j} \text { for } j \in J ; \eta_{\alpha}, \eta_{j} \geq 0, j \in J\right\} .
\end{aligned}
$$

(III) There exist equalities only in (1.4) for indices $j \in J$ (possibly empty) and (1.6). Then,

$$
\begin{aligned}
\mathcal{N}_{\boldsymbol{\Lambda}(\theta)}(\boldsymbol{\lambda})=\left\{\mathbf{k} \in \mathbb{R}^{d}: k_{j}=\frac{\eta_{\beta}}{\left(\beta-\lambda_{j}\right)^{2}} \text { for } j \notin J,\right. \\
\left.\quad k_{j}=\frac{\eta_{\beta}}{\left(\beta-\lambda_{\theta}^{+}\right)^{2}}+\eta_{j} \text { for } j \in J ; \eta_{\beta}, \eta_{j} \geq 0, j \in J\right\} .
\end{aligned}
$$

(IV) There exist equalities in (1.5) and (1.6) (this implies that (1.4) holds for the set $J$ of $d-1$ indices $j$, and the remaining $\lambda_{j}$ is equal to $\left.\lambda_{\theta}^{-}\right)$. Then,

$$
\begin{aligned}
& \mathcal{N}_{\boldsymbol{\Lambda}(\theta)}(\boldsymbol{\lambda})=\left\{\mathbf{k} \in \mathbb{R}^{d}: k_{j}=-\frac{\eta_{\alpha}}{\left(\lambda_{j}-\alpha\right)^{2}}+\frac{\eta_{\beta}}{\left(\beta-\lambda_{j}\right)^{2}} \text { for } j \notin J,\right. \\
& k_{j}=-\frac{\eta_{\alpha}}{\left(\lambda_{\theta}^{+}-\alpha\right)^{2}}+\frac{\eta_{\beta}}{\left(\beta-\lambda_{\theta}^{+}\right)^{2}}+\eta_{j} \text { for } j \in J \text {; } \\
& \left.\eta_{\alpha}, \eta_{\beta}, \eta_{j} \geq 0, j \in J\right\} \text {. }
\end{aligned}
$$

\section{Proof of Theorem 2.1}

Thanks to convexity of $\mathscr{H}$, the boundary points can be characterised as points in which for some vectors $\mathbf{w}_{1}, \ldots, \mathbf{w}_{m} \in \mathbb{R}^{d}$,

$$
\varphi(\mathbf{A}):=\sum_{i=1}^{m} \mathbf{A} \mathbf{a}_{i} \cdot \mathbf{w}_{i}
$$

attains its minimum on $\mathscr{K}(\theta)$.

Let $\left(\mathbf{e}_{1}, \ldots, \mathbf{e}_{m}\right)$ be the orthonormal basis for vector space $\operatorname{Span}\left(\left\{\mathbf{a}_{1}, \ldots, \mathbf{a}_{m}\right\}\right) \leq \mathbb{R}^{d}$. If $\mathbf{a}_{i}=\sum_{j=1}^{m} \gamma_{j}^{i} \mathbf{e}_{j}$ is the representation of vector $\mathbf{a}_{i}$ in this basis, we can write

$$
\varphi(\mathbf{A})=\sum_{j=1}^{m} \mathbf{A e}_{j} \cdot \mathbf{v}_{j},
$$


where $\mathbf{v}_{j}=\sum_{i=1}^{m} \gamma_{j}^{i} \mathbf{w}_{i}, j=1, \ldots, m$. We will complete the orthonormal set $\left\{\mathbf{e}_{1}, \ldots, \mathbf{e}_{m}\right\}$ to an orthonormal basis $\left\{\mathbf{e}_{1}, \ldots, \mathbf{e}_{d}\right\}$ of $\mathbb{R}^{d}$ and use it in the sequel.

If we introduce a matrix $\mathbf{M}$ with vectors $\mathbf{v}_{1}, \ldots, \mathbf{v}_{m}$ as its first $m$ columns, and null vectors otherwise, then

$$
\min _{\mathbf{A} \in \mathscr{K}(\theta)} \varphi(\mathbf{A})=\min _{\mathbf{A} \in \mathscr{K}(\theta)} \mathbf{A} \cdot \mathbf{M}=\min _{\mathbf{A} \in \mathscr{K}(\theta)} \mathbf{A} \cdot \mathbf{M}_{S},
$$

where $\mathbf{M}_{S}=(1 / 2)\left(\mathbf{M}+\mathbf{M}^{\tau}\right)$ is the symmetric part of matrix $\mathbf{M}$, and - denotes the Euclidean scalar product on $\mathbb{R}^{d \times d}$. The classical von Neumann result says that the rightmost minimum is attained at a matrix $\mathbf{A}$ such that $\mathbf{A}$ and $\mathbf{M}_{S}$ are simultaneously diagonalisable. Therefore, denoting by $\boldsymbol{\lambda}=\left(\lambda_{1}, \ldots, \lambda_{d}\right)$ the eigenvalues of $\mathbf{A}$, and by $\boldsymbol{\mu}=\left(\mu_{1}, \ldots, \mu_{d}\right)$ those of $\mathbf{M}_{S}$, we have

$$
\min _{\mathbf{A} \in \mathscr{K}(\theta)} \varphi(\mathbf{A})=\min _{\lambda \in \boldsymbol{\Lambda}(\theta)} \sum_{n=1}^{d} \lambda_{n} \mu_{n} .
$$

Since the set $\Lambda(\theta)$ is convex, a point $\lambda$ is optimal for the above minimisation problem if and only if the vector $-\boldsymbol{\mu}$ (where $\boldsymbol{\mu}$ is the gradient of the function to be minimised) belongs to the normal cone $\mathcal{N}_{\boldsymbol{\Lambda}(\theta)}(\boldsymbol{\lambda})$ which is described in Lemma 3.1. In Lemma 4.1, we show an interesting property of spectrum $\sigma\left(\mathbf{M}_{S}\right)$.

Lemma 4.1. The spectrum of $M_{S}$ contains at most $m$ positive eigenvalues and at most $m$ negative eigenvalues.

Proof. If eigenvalues of $\mathbf{M}_{S}$ are ordered such that $\mu_{1} \leq \mu_{2} \leq \cdots \leq \mu_{d}$, then the CourantFisher minmax theorem claims that

$$
\mu_{k}=\min _{\substack{V \leq \mathbb{R}^{d} \\ \operatorname{dim} V=k}} \max _{\substack{\mathbf{x} \in V \\\|\mathbf{x}\|=1}} \mathbf{M}_{S} \mathbf{x} \cdot \mathbf{x}
$$

or

$$
\mu_{d-k+1}=\max _{\substack{V \leq \mathbb{R}^{d} \\ \operatorname{dim} V=k}} \min _{\substack{\mathbf{x} \in V \\\|\mathbf{x}\|=1}} \mathbf{M}_{S} \mathbf{x} \cdot \mathbf{x}
$$

Using (4.5), we will prove that $\mu_{m+1} \geq 0$, so at most $m$ eigenvalues $\left(\mu_{1}, \ldots, \mu_{m}\right)$ are negative. By the same idea, from (4.6) it follows that $\mu_{d-m} \leq 0$, implying that at most $m$ eigenvalues are positive.

For any $(m+1)$-dimensional subspace $V$ in $\mathbb{R}^{d}$, we can choose a unit vector $\mathbf{x} \in V$ orthogonal to first $m$ vectors $\mathbf{e}_{1}, \ldots, \mathbf{e}_{m}$ of the orthonormal basis chosen above. Therefore,

$$
\mathbf{M}_{S} \mathbf{x} \cdot \mathbf{x}=\mathbf{M x} \cdot \mathbf{x}=0
$$

because $\mathbf{M x}=\mathbf{0}$, as last $d-m$ columns of $\mathbf{M}$ are null vectors. This implies that

$$
\max _{\substack{\mathbf{x} \in V \\\|\mathbf{x}\|=1}} \mathbf{M}_{S} \mathbf{x} \cdot \mathbf{x} \geq 0
$$


and since $V$ is an arbitrary $(m+1)$-dimensional subspace, taking into account (4.5), it follows that $\mu_{m+1} \geq 0$.

Suppose that $\lambda$ is optimal for (4.4). As in Lemma 3.1, by $J$ we will denote the set of indices $j$ such that the equality $\lambda_{j}=\lambda_{\theta}^{+}$holds. In order to complete the proof of the theorem, it remains to investigate the cases from Lemma 3.1.

(I) If we change $\lambda_{j}$ for only one $j \notin J$ such that (1.5) holds, then for this new $\tilde{\lambda}$, we have $\mathcal{N}_{\boldsymbol{\Lambda}(\theta)}(\boldsymbol{\lambda}) \subseteq \mathcal{N}_{\boldsymbol{\Lambda}(\theta)}(\tilde{\boldsymbol{\lambda}})$, so $\tilde{\boldsymbol{\lambda}}$ is also optimal for (4.4) $\left(-\boldsymbol{\mu} \in \mathcal{N}_{\boldsymbol{\Lambda}(\theta)}(\tilde{\boldsymbol{\lambda}})\right)$. In this way, we obtain an optimal point that corresponds to a sequential laminate with matrix phase $\alpha \mathbf{I}$ and fits in the case II.

(II) By Lemma 3.1, there exist an $\eta_{\alpha} \geq 0$ and $\eta_{j} \geq 0$ for $j \in J$ such that $\mu_{j}=\eta_{\alpha} /\left(\lambda_{j}-\right.$ $\alpha)^{2}$ for $j \notin J$ and $\mu_{j}=\eta_{\alpha} /\left(\lambda_{\theta}^{+}-\alpha\right)^{2}-\eta_{j}$ for $j \in J$. If $\eta_{\alpha}=0$ (in this case, we may assume that $J \neq \varnothing$, as otherwise $\boldsymbol{\mu}=\mathbf{0}$ which implies that any $\boldsymbol{\lambda}$ is optimal), we can pass to another $\tilde{\lambda}$, which is also optimal for (4.4), while it corresponds to a rank-one laminate. Indeed, in this case, we have $\mu_{j}=0$ for $j \notin J$ and $\mu_{j} \leq 0$ otherwise, so if we define $\tilde{\lambda}$ by setting $\tilde{\lambda}_{j}=\lambda_{\theta}^{-}$for only one index $k \notin J$, and $\lambda_{\theta}^{+}$ otherwise, we have $-\boldsymbol{\mu} \in \mathcal{N}_{\Lambda(\theta)}(\tilde{\boldsymbol{\lambda}})$ (see Lemma 3.1, Case IV, a good choice for parameters is $\left.\eta_{\alpha}=\eta_{\beta}=0, \eta_{j}=-\mu_{j}, j \neq k\right)$.

If $\eta_{\alpha}>0$, since Lemma 4.1 claims that $\boldsymbol{\mu}$ has at most $m$ positive components, we must have $\left|J^{c}\right|=d-|J| \leq m$, and Remark 1.1 implies that the optimal $\lambda$ corresponds to a sequential laminate with matrix phase $\alpha \mathbf{I}$ of order at most $m$.

(III) By Lemma 3.1, there exist an $\eta_{\beta} \geq 0$ and $\eta_{j} \geq 0$ for $j \in J$ such that $\mu_{j}$ $=-\eta_{\beta} /\left(\beta-\lambda_{j}\right)^{2}$ for $j \notin J$ and $\mu_{j}=-\eta_{\beta} /\left(\beta-\lambda_{\theta}^{+}\right)^{2}-\eta_{j}$ for $j \in J$.

If $\eta_{\beta}=0$, we can use a similar argument as in II to conclude that a rank-one laminate is also optimal for (4.4).

Otherwise, all components of $\boldsymbol{\mu}$ are negative, which is in contradiction with Lemma 4.1.

(IV) As mentioned in Remark 1.1, in this case, we are already dealing with a rank-one laminate, so there is nothing to prove.

\section{Some consequences and remarks}

Let us first consider the following two questions.

(i) Can the boundary of the set $\mathscr{H}$ be attained by sequential laminates with matrix phase $\beta \mathbf{I}$ ?

(ii) Are there examples in which boundary points of set $\mathscr{H}$ are attained by laminates of rank exactly $m$ (so that Theorem 2.1 cannot be improved)?

Since the case $d=3$ is of main interest in applications, we will deal with this case more thoroughly and present some examples that will answer these questions. Anyway, the presented examples can be easily modified to any dimension $d \geq 3$. For the exact computation of (4.4) in the case $m=d=2$, see [1, Lemma 3.2.17]. In three dimensions, the case $k=1$ is studied in Lemma 1.2 , so the only case left is $k=2$. As shown at the beginning of the proof of Theorem 2.1, the case of linearly independent vectors $\mathbf{a}_{1}$ and $\mathbf{a}_{2}$ reduces to the same problem for orthonormal vectors $\mathbf{e}_{1}, \mathbf{e}_{2}$. Therefore, without loss of generality, we can start with $\mathbf{a}_{1}=(1,0,0)^{\tau}, \mathbf{a}_{2}=(0,1,0)^{\tau}$, whence $\mathbf{w}_{1}=\mathbf{v}_{1}$ and $\mathbf{w}_{2}=\mathbf{v}_{2}$. 


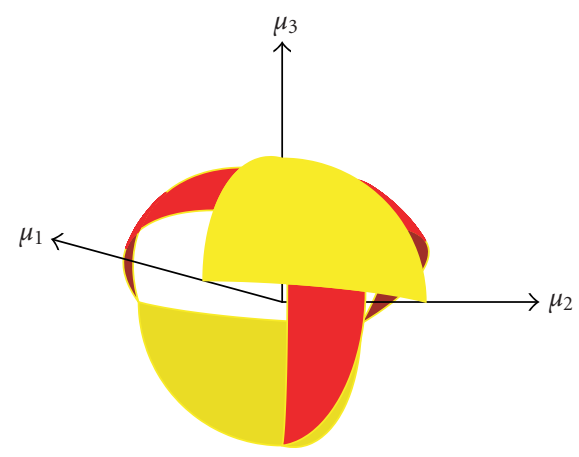

Figure 5.1. Optimal microstructures for given unit vector $\mu$ : red represents rank-two laminates, yellow means cannot occur, else represents rank-one laminates.

In the proof of Theorem 2.1, we showed that the question of determining the boundary of set $\mathscr{H}$ reduces to the minimisation of

$$
\lambda \longmapsto \sum_{i=1}^{3} \lambda_{i} \mu_{i}
$$

over set $\Lambda(\theta)$ given by (1.4)-(1.6). Thanks to the explicit description of normal cone $\mathcal{N}_{\boldsymbol{\Lambda}(\theta)}(\boldsymbol{\lambda})$ at $\boldsymbol{\lambda} \in \boldsymbol{\Lambda}(\theta)$ given in Lemma 3.1, for given vector $\boldsymbol{\mu}=\left(\mu_{1}, \mu_{2}, \mu_{3}\right)$, we can easily determine whether the optimal microstructure for (4.4) is a rank-one or rank-two laminate. Since the optimal $\lambda \in \Lambda(\theta)$ for (5.1) does not change if one multiplies $\boldsymbol{\mu}$ by some positive scalar, it is enough to consider unit vectors $\boldsymbol{\mu}$ (if $\boldsymbol{\mu}$ is the null vector, any $\boldsymbol{\lambda}$ is optimal).

Moreover, by the construction in the proof of Theorem 2.1, not all unit vectors $\boldsymbol{\mu}$ should be considered: we start from vectors $\mathbf{v}_{1}, \mathbf{v}_{2} \in \mathbb{R}^{3}$, construct matrix $\mathbf{M}$ with columns $\mathbf{v}_{1}, \mathbf{v}_{2}$ and a zero vector, and normalise the vector formed by eigenvalues for the symmetric part of $\mathbf{M}$ to obtain $\boldsymbol{\mu}$. That leads us to Figure 5.1. Yellow regions (open sets) correspond to all such $\boldsymbol{\mu}$ that have all components of the same sign, which cannot be attained as claimed in Lemma 4.1. In spherical coordinates, they are written as $\varphi, \vartheta \in\langle 0, \pi / 2\rangle$ or $\varphi \in\langle\pi, 3 \pi / 2\rangle, \vartheta \in\langle\pi / 2, \pi\rangle$. Furthermore, in the same picture, the directions $\boldsymbol{\mu}$ for which the corresponding optimal microstructure is a rank-two laminate with matrix material $\alpha \mathbf{I}$ depicted by red. One of the regions is described by (the others are symmetric) $\varphi \in\left\langle\varphi_{0}, \pi / 2-\varphi_{0}\right\rangle, \vartheta \in\langle\pi / 2, \pi\rangle$, with $\varphi_{0}=\arctan \left(\lambda_{\theta}^{-}-\alpha\right)^{2} /\left(\lambda_{\theta}^{+}-\alpha\right)^{2}$. The remaining part of the sphere corresponds to rank-one laminates.

Now, for the answers to both questions stated above, it is enough to show examples of $\mathbf{v}_{1}$ and $\mathbf{v}_{2}$ with corresponding $\boldsymbol{\mu}$ belonging to the red region in Figure 5.1. For the first one, we took $\mathbf{v}_{1}=(1,0,0)^{\tau}$ and $\mathbf{v}_{2}=(0, t, 0)^{\tau}$ with parameter $t \in[0,1]$, so that $\boldsymbol{\mu}$ is a unit vector in direction $(1, t, 0)^{\tau}$ (Figure 5.2(a)). If $t=0$, we know (Lemma 1.2) that a rankone laminate is optimal (point A). For $t=\left(\lambda_{\theta}^{-}-\alpha\right)^{2} /\left(\lambda_{\theta}^{+}-\alpha\right)^{2}$, the corresponding $\boldsymbol{\mu}$ enters the red region, and stays there until $t=1$. The other example presented in Figure 5.2(b) is $\mathbf{v}_{1}=(1,0,0)^{\tau}, \mathbf{v}_{2}=(0,4 t-1,3-4 t)^{\tau}$, for $t \in[0,1]$. 


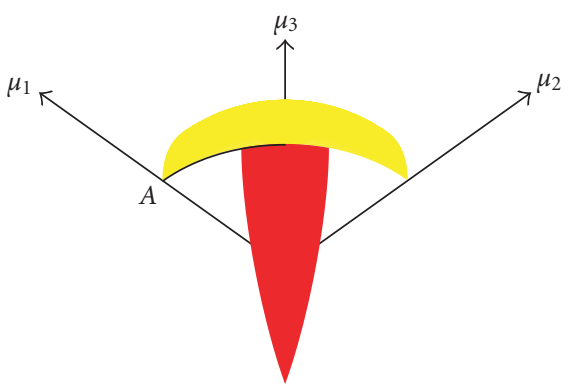

(a)

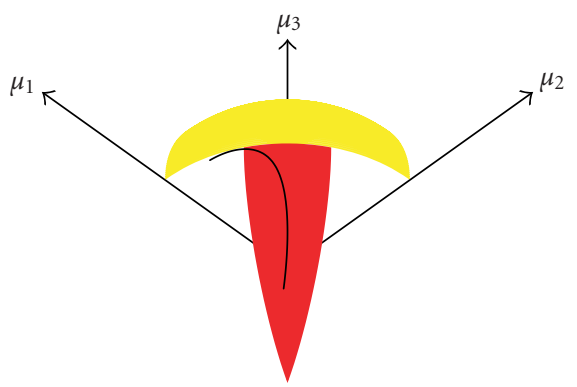

(b)

Figure 5.2. (a) and (b) are two examples of optimal microstructures.

Finally, we would like to compare our result to [1, Theorem 3.2.14]. The latter gives optimality conditions for optimal design problems with multiple-state equations, which are the basis for numerical treatment of the problem. As discussed in [1, Remark 5.1.2], the fact that an optimal microstructure can be chosen among rank- $d$ laminates simplifies the calculation and makes it feasible.

The assumption $m<d$ turns out to have significant consequences for the calculation of the rank of optimal sequential laminate. Of interest is the fact that sequential laminates with matrix phase $\alpha \mathbf{I}$ are preferred to those with matrix phase $\beta \mathbf{I}(\alpha<\beta)$. In the case $m=d$, the result of [1, Theorem 3.2.14] cannot be improved: the boundary of the set $\mathscr{H}$ is attained by sequential laminates of rank $d$, with matrix phase $\alpha \mathbf{I}$ or $\beta \mathbf{I}$.

\section{A numerical example}

Let us demonstrate the significance of our result for engineering applications by a simple numerical example. For simplicity, we consider a problem of a heated/cooled cube $\Omega=$ $[-1,1]^{3}$ in two regimes of operation; differing in the source term (cf. (1.1))

$$
f_{1}(\mathbf{x})=\left\{\begin{array}{ll}
0, & x_{1}<0, \\
-3, & x_{1} \geq 0,
\end{array} \quad f_{2}(\mathbf{x})= \begin{cases}2, & x_{1}<0 \\
0, & x_{1} \geq 0\end{cases}\right.
$$




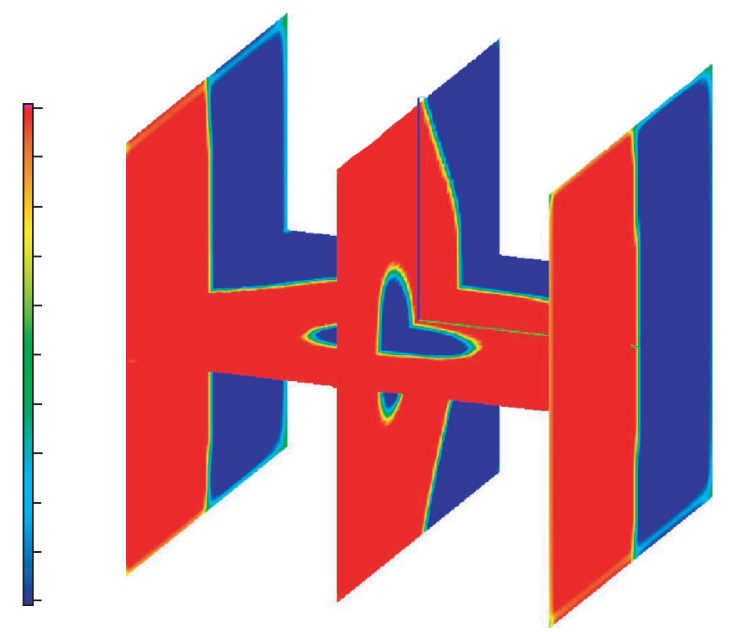

Figure 6.1. Optimal distribution of materials: $\theta=0$ corresponds to $\beta \mathbf{I}$ and $\theta=1$ to $\alpha \mathbf{I}$ (only four cut-planes of the cube are shown).

In both cases, the boundary of the cube is kept at the fixed temperature-set at zero. We will take $\alpha=1$ and $\beta=2$. The cost functional is given by

$$
J(\theta, \mathbf{A})=\int_{\Omega}\left(u_{1}+u_{2}\right) d \mathbf{x}
$$

By the result of Allaire [1, Remark 5.1.2], instead of considering the whole $\mathscr{Y}(\theta)$, one should optimise only over its hyperboloid bounding surfaces shown in Figure 1.2(a). However, our result shows that it is enough to optimise over three bold curves in the same figure. Because of symmetry, we can take only one of them (say the one lying in the upper face of the cube) where $\lambda_{3}=\lambda_{\theta}^{+}$and $\left(\lambda_{1}, \lambda_{2}\right)$ belongs to a curve shown in Figure 1.2(b) (in fact, only a half of the curve matters, as shown in the picture). A point on this curve is parametrised by $\epsilon \in[0,1]$, while an $\mathbf{A}$ is determined by its eigenvalues $\left(\lambda_{1}, \lambda_{2}, \lambda_{3}\right)$ given through parameters $\epsilon$ and $\theta$ and a rotation described in terms of Euler angles $\varphi, \vartheta$, and $\psi$.

In this way, the cost $J$ is determined by five parameters: $\theta, \epsilon, \varphi, \vartheta$, and $\psi$ (functions of $\mathbf{x} \in \Omega$ ). For its optimisation, we tested several known methods: steepest descent and conjugated gradients, with the Newton method for line search. For these methods, we need the gradient of the cost function with respect to $\theta, \epsilon, \varphi, \vartheta$, and $\psi$, which is obtained by straightforward, though tedious computation, in a similar vein as in [1, Section 5.1.2], where this is done in two dimensions for one-state equation.

In each optimisation step, we have to solve state equations for both $u_{1}$ and $u_{2}$, as well as two adjoint boundary value problems needed for the calculation of the gradient. We used deal. II library for finite elements [4], and run the program up to $64 \times 64 \times 64$ cubic mesh, with bilinear elements and order-two Gaussian quadratures, which took several hours to complete on a PC computer.

In Figure 6.1, we present the solution $\theta$ obtained by Fletcher-Reeves conjugated gradient method, with convergence history in Figure 6.2. We tested it with different initial 
N. Antonić and M. Vrdoljak 13

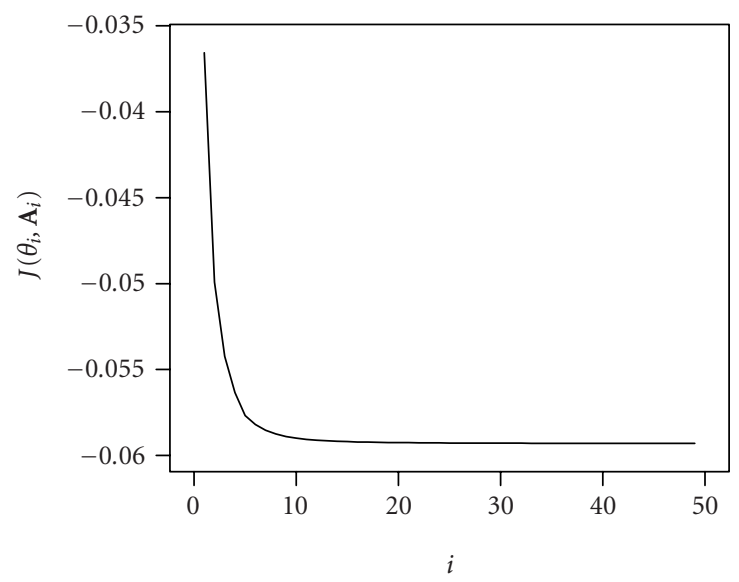

(a)

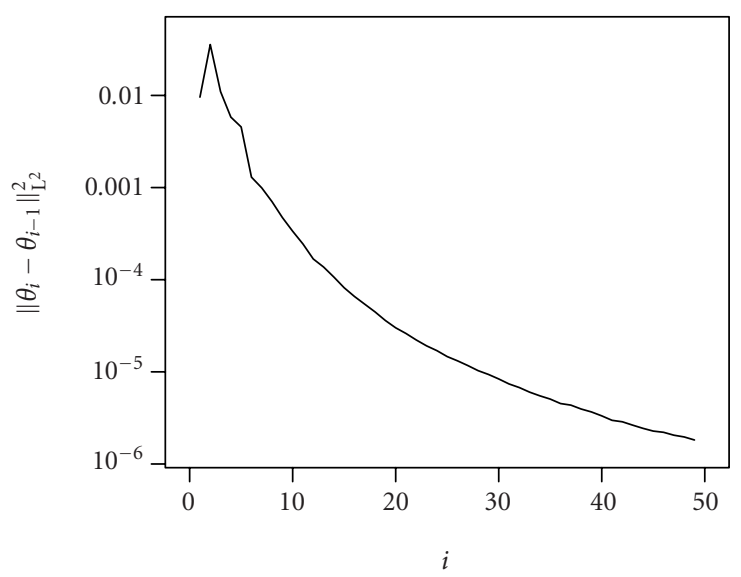

(b)

Figure 6.2. Convergence history: (a) cost functional and (b) $\left\|\theta_{i}-\theta_{i-1}\right\|_{\mathrm{L}^{2}}^{2}$, in terms of the iteration number $i$.

parameters, and obtained approximately the same result. The steepest descent method and Polak-Robiere conjugated gradient method also gave similar results.

For further applications, one should add a constraint on the amount of material $\alpha \mathbf{I}$ : $\int_{\Omega} \theta d \mathbf{x} \leq V_{\alpha}$, which can be treated by Lagrange multipliers in a standard manner $[1,5]$.

\section{Acknowledgments}

This research was supported by Croatian Ministry of Science and Technology through Grant 0037101. The numerical work was performed on computers in Laboratory for Advanced Computing at Mathematics Department, University of Zagreb. 


\section{Multiple-state optimal design problems}

\section{References}

[1] G. Allaire, Shape Optimization by the Homogenization Method, Applied Mathematical Sciences, vol. 146, Springer, New York, 2002.

[2] N. Antonić and M. Vrdoljak, On some projections of homogenised coefficients for stationary diffusion equation, Proceedings in Applied mathematics and Mechanics 3 (2003), 440-441.

[3] _ On some properties of homogenised coefficients for stationary diffusion problem, Proceedings of the Conference on Applied Mathematics and Scientific Computing, Springer, 2005, pp. $121-130$.

[4] W. Bangerth, G. Kanschat, R. Hartmann, et al., The deal.II library, http://www.dealii.org/.

[5] M. P. Bendsøe and O. Sigmund, Topology Optimization. Theory, Methods and Applications, Springer, Berlin, 2003.

[6] A. Cherkaev and R. Kohn (eds.), Topics in the Mathematical Modelling of Composite Materials, Progress in Nonlinear Differential Equations and Their Applications, vol. 31, Birkhäuser Boston, Massachusetts, 1997.

[7] F. H. Clarke, Optimization and Nonsmooth Analysis, 2nd ed., Classics in Applied Mathematics, vol. 5, SIAM, Pennsylvania, 1990.

[8] F. Murat and L. Tartar, Calcul des variations et homogénéisation [Calculus of variations and homogenization], Homogenization Methods: Theory and Applications in Physics (Bréau-sansNappe, 1983), Collect. Dir. Études Rech. Élec. France, vol. 57, Eyrolles, Paris, 1985, pp. 319-369.

[9] R. T. Rockafellar, Convex Analysis, Princeton Mathematical Series, no. 28, Princeton University Press, New Jersey, 1970.

[10] L. Tartar, Estimations fines des coefficients homogénéisés [Fine estimates of homogenized coefficients], Ennio De Giorgi Colloquium (Paris, 1983) (P. Krée, ed.), Res. Notes in Math., vol. 125, Pitman, Massachusetts, 1985, pp. 168-187.

[11] _ Remarks on the homogenization method in optimal design methods, Homogenization and Applications to Material Sciences (Nice, 1995), GAKUTO Internat. Ser. Math. Sci. Appl., vol. 9, Gakkōtosho, Tokyo, 1995, pp. 393-412.

[12] _ An introduction to the homogenization method in optimal design, Optimal Shape Design (Tróia, 1998) (A. Cellina and A. Ornelas, eds.), Lecture Notes in Math., vol. 1740, Springer, Berlin, 2000, pp. 47-156.

Nenad Antonić: Department of Mathematics, University of Zagreb, Bijenička cesta 30, 10000 Zagreb, Croatia

E-mail address: nenad@math.hr

Marko Vrdoljak: Department of Mathematics, University of Zagreb, Bijenička cesta 30, 10000 Zagreb, Croatia

E-mail address: marko@math.hr 


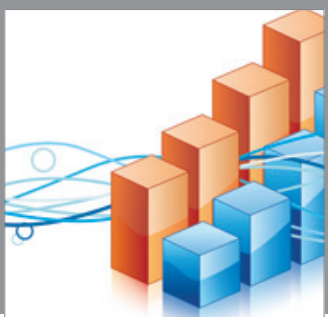

Advances in

Operations Research

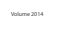

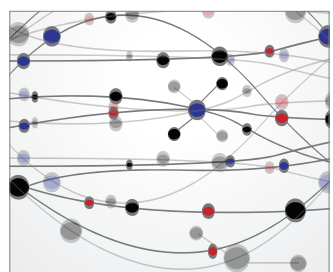

\section{The Scientific} World Journal
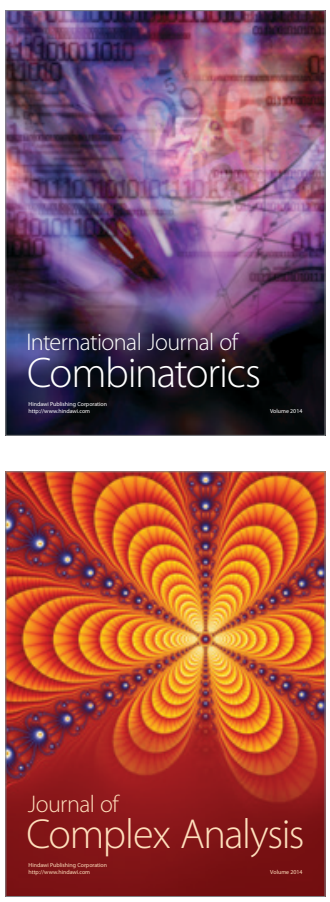

International Journal of

Mathematics and

Mathematical

Sciences
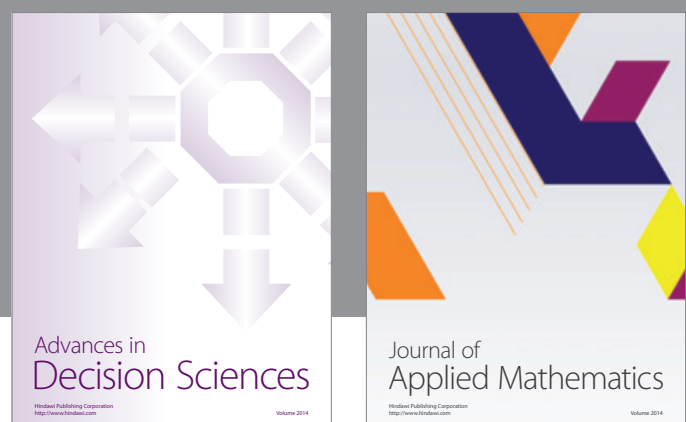

Journal of

Applied Mathematics
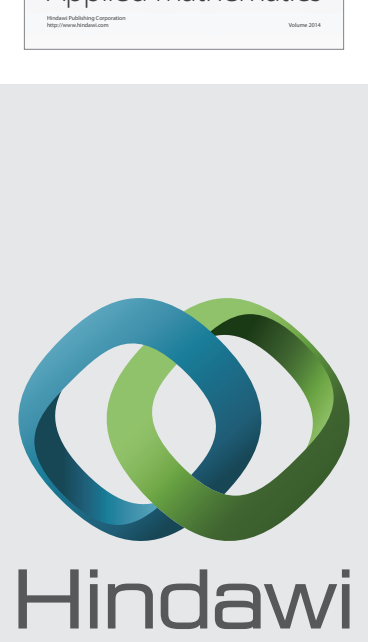

Submit your manuscripts at http://www.hindawi.com
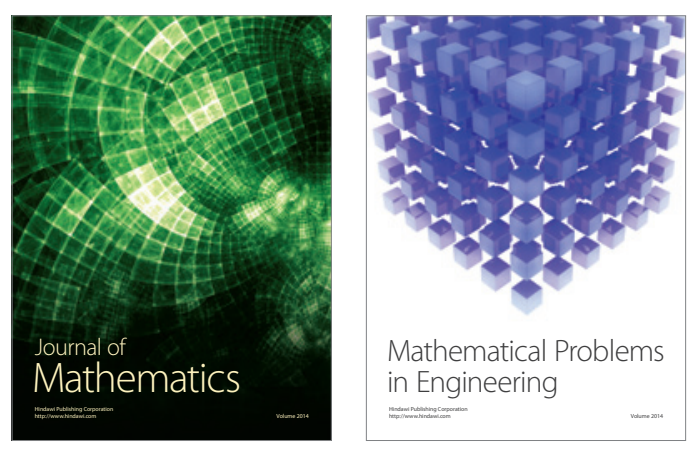

Mathematical Problems in Engineering
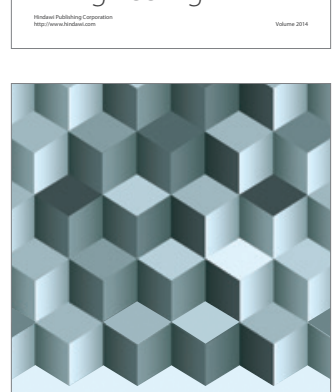

Journal of

Function Spaces
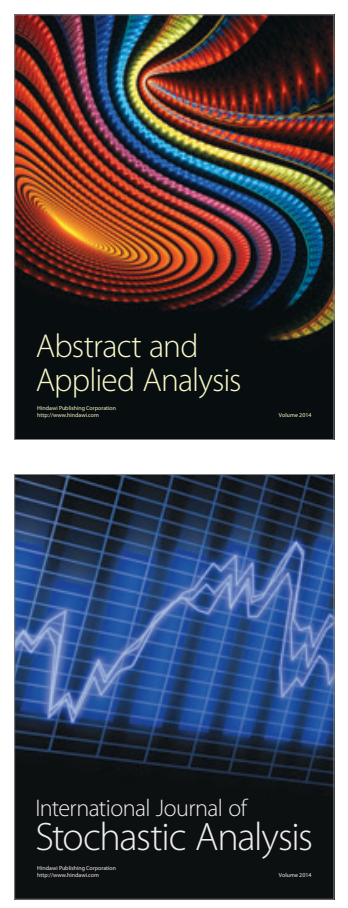

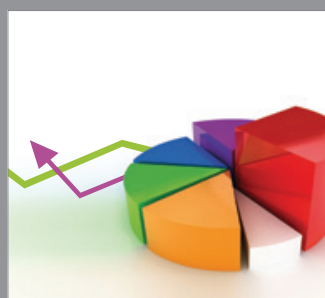

ournal of

Probability and Statistics

Promensencen
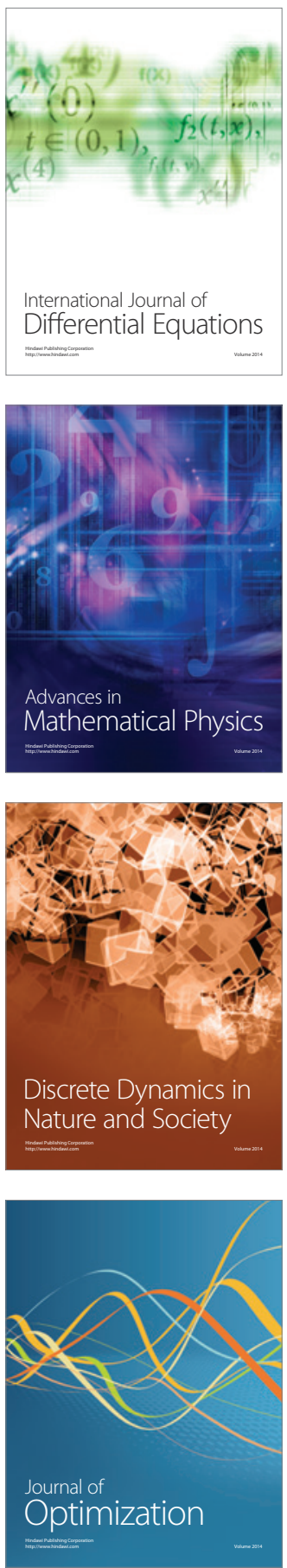